\title{
Effect of incorporation of peanut and sesame oils and their epoxides on the structure of Poly Vinyl Chloride
}

\author{
L.Karunanayake* and P.N.J.Fernando \\ Department of Chemistry, University of Sri Jayewardenepura, Gangodawila, Nugegoda.
}

\begin{abstract}
Epoxidised soybean oil (ESO) is widely used as a plasticizer for Poly Vinyl Chloride (PVC). The interactions of the sesame oil and the peanut oil and their epoxides with PVC were investigated in this study. The epoxidation of the oils were carried out in sitn using the peracetic acid method. Epoxidation was confirmed using $\mathrm{H}^{\prime}$ NMR and ${ }^{13} \mathrm{C}$ NMR spectra. The interaction of oils and their epoxides with PVC were evaluated using FT-IR (Fourier Transform Infrared) spectra. Soybean oil was treated as the benchmark. According to the FT-IR analysis, the oils and their epoxy derivatives were found to interact with PVC molecules. These interactions solubilize the PVC in the plasticisers and hence make them compatible with PVC. It is also shown that these molecules break the polymer-polymer interactions and mask the interactive sites of the PVC polymers. In comparison to soybean oil, molecular interactions of sesame oil with PVC is better. However, peanut oil and soybean oil show better molecular interactions below 10 phr level, while sesame oil shows maximum interactions around 50 phr.
\end{abstract}

Key words: Epoxidisation, peanut oil, plasticizer, PVC, sesame oil

\section{INTRODUCTION}

Plasticizers are low melting solids or high boiling organic materials added to plastics to improve or to impart better properties. ${ }^{1}$ Addition of plasticizers is essential to facilitate processing and to obtain desired flexibility. Plasticization occurs when polar groups of the plasticizer interacts with polar groups of the polymer, reducing polymer-polymer interaction. Plasticizer molecule requires a polar group to facilitate interactions with the polymer and a non-polar chain to reduce polymer-polymer interaction penetrating between polymer molecules. ${ }^{2}$ Most commonly used plasticizers are phthalates such as Dioctyl phthalate (DOP) ${ }^{3}$ and adipates such as di-2-ethylhexyladipate (DEHA). ${ }^{4}$
In addition, the use of Epoxidized Soybean Oil (ESO) as a plasticizer or as a secondary plasticizer for PVC is well known and it is a commercially available material produced by several manufacturers. ${ }^{5}$

Use of derivatives of natural oils as plasticizers for PVC has been studied since the mid sixties. Epoxidized triglycerides and their esters have been reported as excellent substitutes for conventional phthalate and adipate type plasticizers. This is due to the fact that apart from being good plasticizers, they also impart remarkable improvement in both heat and light stability of PVC resins due to the presence of the oxirane rings. ${ }^{6}$ While epoxidized soybean oil is offered as a commercial product, several other epoxidized natural oils such as linseed, rapeseed, olive, corn and cotton seed have been reported to act as good plasticizers/ stabilizers for PVC. ${ }^{6}$ The use of Epoxidized Linseed Oil (ELO) together with $\mathrm{ESO}$ and some other conventional plasticizers in a study where the dependence of properties of ion exchange resins on the level of plasticizer content have been reported.' Further, the use of rapeseed methyl ester (RME) as a plasticizer for PVC has been investigated. It has been reported that RME can replace about $50 \%$ of phthalate plasticizers used in PVC web applications. ${ }^{8}$ Further, the use of ESO alongside with conventional plasticizer for various studies such as effect of ESO as secondary stabilizer in rigid vinyl for exterior applications ${ }^{9}$, synergistic effect of ESO and zinc stearate on PVC plastigels made using DOP,$^{10}$ concentration dependency of plasticizer on interaction parameters between PVC and plasticizers (DOP and ESO) ${ }^{2}$, etc. has been reported. 
Hence, in this research study an attempt was made to find the feasibility of the use of two vegetable oils, namely peanut oil and sesame oil, and their derivatives as plasticizers /stabilisers for PVC. Being the most proven vegetable oil derivative type, the epoxy derivatives of the two vegetable oils were investigated. In this study, wellestablished vegetable oil based plasticizer/stabiliser soybean oil was used as the benchmark to compare the two other vegetable oils.

M. Theodorou, B. Jasse ${ }^{12}$ have reported that FT-IR (Fourier-Transform Infrared) spectroscopy can be used to detect the conformational changes induced by plasticization in atactic PVC. They have explained that the amount of short trans syndiotactic sequences decrease upon plasticization and the change depends on the amount and kind of plasticizer. They have further added that the difference in spectra emphasize changes in the distribution of gauche defects in the chains as a function of temperature in plasticized PVC films.

However, as a preliminary study, molecular interactions between vegetable oils and their derivatives with PVC molecules were studied using the FT-IR spectroscopy. These spectral changes were used to gauge inter molecular interactions between PVC and oil based plasticizers.

As a large quantity of PVC products produced is consumed by the food industry as packing materials, there is a growing concern about the toxicity and the migration of small plasticizer molecules from the polymeric packaging material to foodstuff. ${ }^{4}$ Further, it has been reported that the use of plasticized PVC materials such as PVC flooring in indoor applications emit phthalate basad nlast ${ }^{\circ}$ : The situation is worst when in is curne to some of the medical applications such as blood storage bags ${ }^{13}$, tubing for catheters and toys made of flexible PVC. ${ }^{3}$ Further, it has been reported that the attack of micro-organisms and fungi on DOP in PVC floor tiles emits toxic materials to air. ${ }^{14}$ The use of natural oils to replace the conventional phthalate and adipate based plasticizers can also address the issue of toxicity.

The main objective of this research was to investigate the chemical interaction of vegetable oils and their epoxides with PVC in order to find their suitability as plasticizer.

\section{METHODS AND MATERIALS}

FT-IR Analysis of pure oil plasticizer: PVC and pure vegetable oils were mixed to obtain the mixtures with the oil composition $5 \mathrm{phr}, 10 \mathrm{phr}, 15 \mathrm{phr}, 20 \mathrm{phr}, 30 \mathrm{phr}$, $40 \mathrm{phr}, 50 \mathrm{phr}, 60 \mathrm{phr}$, and $70 \mathrm{phr}$. Then the oil and PVC samples were dissolved in $50 \mathrm{~cm}^{3}$ of MEK by warming to about $50-60^{\circ} \mathrm{C}$. This procedure was used to obtain the solutions with different oil concentrations.

To obtain the spectrum of the sample, one drop from the prepared solution was placed on $\mathrm{NaCl}$ plate using a Pasteur pipette. The drop was spread over the plate by turning the plate. Then, the solvent was evaporated by blowing a stream of hot air using a Hairdryer. The thickness of the film was increased by repeating the procedure three times. The sample containing $\mathrm{NaCl}$ plate was mounted on the sample holder and the spectrum was obtained.

FT-IR spectra of pure oils: Two drops of pure oil were placed at the centre of a clean and dry $\mathrm{NaCl}$ plate using a Pasteur pipette. A second $\mathrm{NaCl}$ plate was placed on top of the first to sandwich the liquid to a thin film. Then the two plates with the oil were mounted in a sample holder. The sample holder was placed in the sample beam of the FT-IR instrument and the spectrum was recorded.

FT-IR of pure PVC powder: About $2 \mathrm{mg}$ of PVC sample was ground into fine powder. About $200-300 \mathrm{mg}$ of spectroscopic grade Potassium Bromide ( $\mathrm{KBr}$ ) was mixed thoroughly with the PVC sample. Then a disk made of $\mathrm{KBr}$ and pure PVC was made in a press and placed in the IR beam of the spectrometer. The spectrum was obtained.

Iodine value test for pure oils: Samples of soybean (131.1 $\mathrm{mg})$, peanut (200.6 mg) and sesame oils (130.9 mg) were weighed into three different reagent bottles. Oils were dissolved in $15 \mathrm{~cm}^{3}$ of carbon tetrachloride. Exactly 25.00 $\mathrm{cm}^{3}$ of Wij's solution was added to each bottle and closed. The bottles were shaken gently and kept in the dark for 1 h.

Exactly $20.00 \mathrm{~cm}^{3}$ of $10 \%$ Potassium Iodide (KI) solution was added to each bottle after $1 \mathrm{~h}$. About 100 $\mathrm{cm}^{3}$ distilled water was also added to the each bottle. Then this mixture was titrated with standardized $0.1 \mathrm{M}$ $\mathrm{Na}_{2} \mathrm{~S}_{2} \mathrm{O}_{3}$

This procedure was followed for sesame oil, peanut oil and soybean oil separately. A blank test was also carried without adding oil.

Epoxidation using resin as a catalyst: The amount of unsaturation was calculated as a molarity. The amount of reagents that should be added to the reactor was calculated according to the molar ratio of, oil containing 1.0 
mole unsaturation: 0.55 moles of glacial acetic acid: $12 \%$ dry resin based on the weight of the oil: 1.1 moles of $\mathrm{H}_{2} \mathrm{O}_{2}$.

The sample of oil, acetic acid and the resin were added to the flask. Then the mixture was heated to 50-60 ${ }^{\circ} \mathrm{C}$. The $\mathrm{H}_{2} \mathrm{O}_{2}$ was added to the mixture over a $10 \mathrm{~min}$ time period while stirring. The mixture was subjected to constant stirring, at a temperature between $50-60^{\circ} \mathrm{C}$ for 4 h. After $4 \mathrm{~h}, 50 \mathrm{~cm}^{3}$ of distilled water was added to the mixture. The two liquid phases were separated from semisolid resin at the bottom by decantation. The two liquid phases were separated using a separation funnel. The oil phase separated was dissolved in $30 \mathrm{~cm}^{3}$ of diethyl ether and anhydrous $\mathrm{Na}_{2} \mathrm{SO}_{4}$ was added and kept for about $1 \mathrm{~h}$. Then, the clear solution was separated by decantation. The solvent, diethyl ether, was removed by rotary evaporation. The viscous liquid of oil epoxide was obtained.

The confirmation tests for the epoxidation: IR (Infra Red) spectroscopy and NMR (Nuclear Magnetic Resonance) techniques.

FT-IR analysis: FT-IR for the epoxide was carried out according to the liquid method.

${ }^{1} H$ NMR and ${ }^{13} \mathrm{C} N M R$ analysis: About $25 \mathrm{mg}$ of the product was dissolved in $\mathrm{CDCl}_{3}$ solvent. The solution was placed in the NMR sample tube. The tube was sealed and placed in the NMR instrument. The ${ }^{13} \mathrm{C}$ NMR and ${ }^{1} \mathrm{H}$ NMR spectra were recorded.

Determination extent of epoxidation: The extent of epoxidation was calculated using the percentage reduction of Iodine value.

FT-IR analysis of the epoxdised plasticizer: The FT-IR analysis of each produced epoxidised material was carried out.

\section{RESULTS}

\section{Iodine values of oils}

Iodine values of $118.5,94.8$ and 102.5 were found for soybean, peanut and sesame oils, respectively.

\section{Epoxidation}

The epoxidized products that were obtained were lighter in colour and higher in viscosity compared to the pure oils.

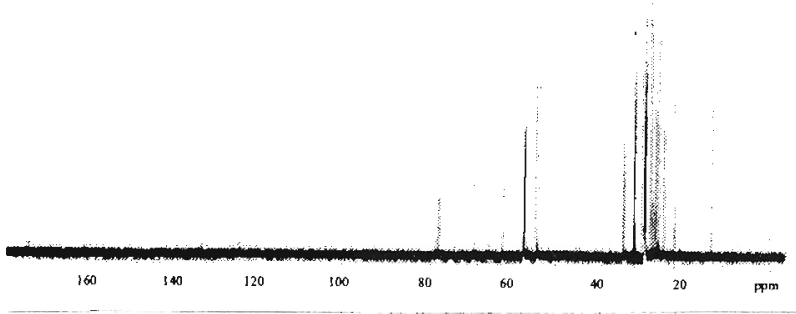

Figure 1: ${ }^{13} \mathrm{C}$ NMR spectrum of epoxidized soybean oil.

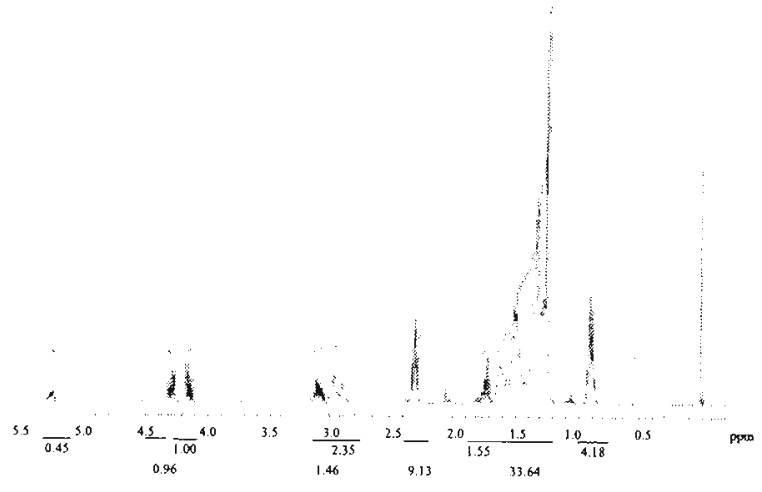

Figure 2: ${ }^{1} \mathrm{H}$ NMR spectrum of epoxidized soybean oil.

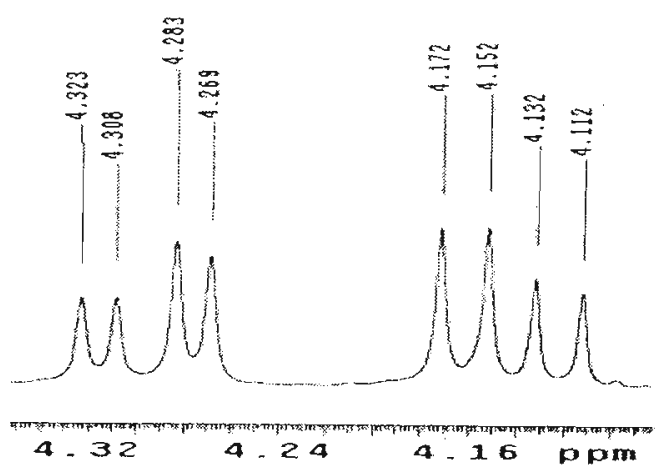

Figure 3: Splitting pattern of the epoxy proton.

\section{Confirmation of epoxide}

\section{FT-IR spectra}

Although, asymmetrical stretching of the (C-C) in the epoxy ring during the contraction of the $(\mathrm{C}-\mathrm{O})$ bond (950-810 $\left.\mathrm{cm}^{-1}\right)$ can be observed, $(\mathrm{C}-\mathrm{H})$ stretching of the 
epoxy ring (3050-2990 $\mathrm{cm}^{-1}$ ) and all ring bond stretching and contracting in phase (near $1250 \mathrm{~cm}^{-1}$ ) cannot be observed clearly due to the overlapping with other peaks.

\section{DISCUSSION}

Use of Epoxidized soybean oil as a plasticizer for PVC is well recorded. ${ }^{2,5,7,10}$ Further, it has been reported that other oils that have shown plasticising effects on PVC, such as sunflower oil, linseed oil, castor oil, olive oil, rape seed oil, cotton seed oil and corn oil have chemical composition similar to that of soybean oil. ${ }^{6}$ Therefore, oils which have a similar chemical composition to that of soybean oil, were selected in this research study (Table 2) and their performance compared with that of soybean oil.

Table 1: Extent of epoxidation achieved for three oils

\begin{tabular}{lrrr}
\hline Type of sample & Iodine value Extent of epoxidation (\%) \\
\cline { 3 - 4 } & & Expected & Actual \\
\hline soybean oil & 11.48 & 100 & 90.3 \\
soybean oil & 62.44 & 50 & 47.3 \\
peanut oil & 9.95 & 100 & 89.5 \\
peanut oil & 52.85 & 50 & 44.5 \\
sesame oil & 3.86 & 100 & 96.2 \\
sesame oil & 53.47 & 50 & 47.8 \\
\hline
\end{tabular}

Table 2: Comparison of features of soybean, sesame and peanut oils

\begin{tabular}{lccc}
\hline \multirow{2}{*}{ Chemical } & \multicolumn{3}{c}{ Type of oil } \\
\cline { 3 - 4 } Property & Soybean oil & Peanut oil & Sesame oil \\
\hline Oleic acid & $23.3 \%$ & $47 \%$ & $40 \%$ \\
Linoleic acid & $53.7 \%$ & $32 \%$ & $43 \%$ \\
Linolenic acid & $7.6 \%$ & small & small \\
Total unsaturation & $85 \%$ & $80 \%$ & $83 \%$ \\
Total saturation & $15 \%$ & $20 \%$ & $17 \%$ \\
\hline
\end{tabular}

Iodine values

The iodine values obtained were found to be lower than the reported values for the oils. ${ }^{16}$ As the oils obtained were commercial products from the local market, the lower Iodine values may have been due to the oxidation of the double bond in the oil by atmospheric oxygen ${ }^{18}$ and the impurities in the oil. The Iodine values of the oils were used for the determination of the number of

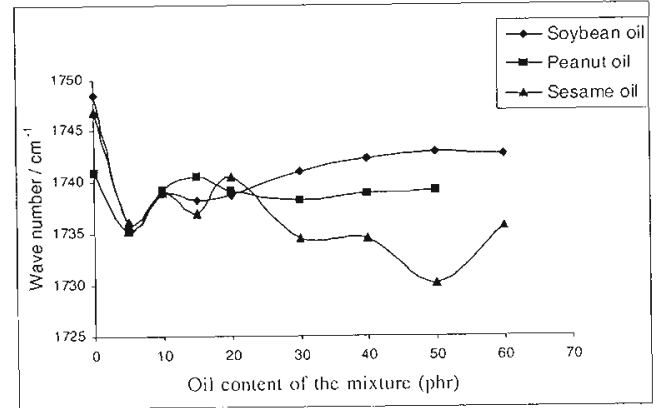

Figure 4: Change of the $(\mathrm{C}=0)$ peaks with different contents of pure oil.

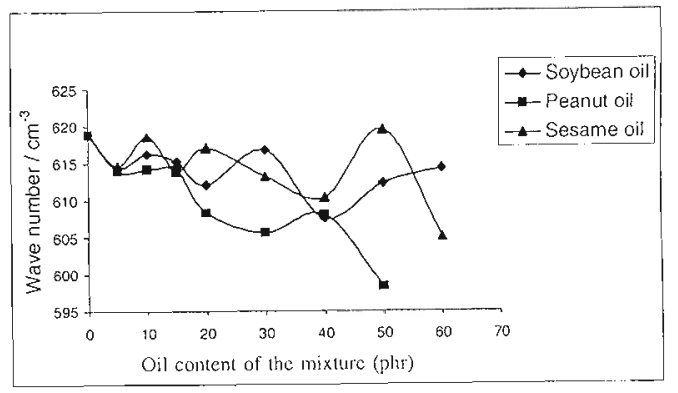

Figure 5: Change in the (C-Cl) of the PVC in pPVC mixture

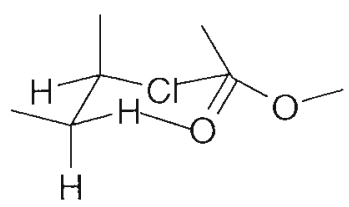

Figure 6: the six centered interaction between oil and PVC.

FT-IR spectra of the $100 \%$ epoxidized oil derivatives

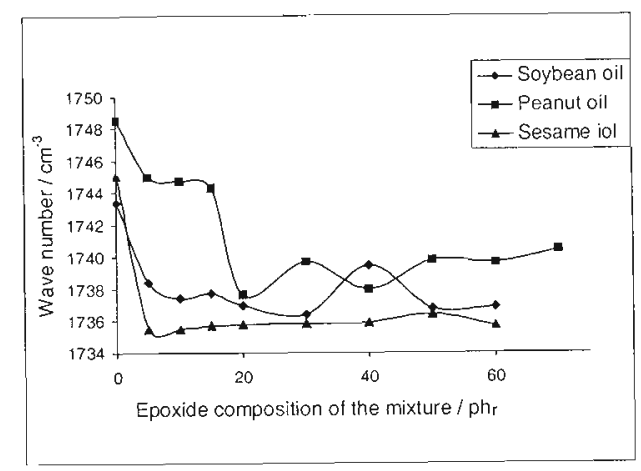

Figure 7: Change in position of the $(\mathrm{C}=\mathrm{O})$ of the epoxidized oil.

double bonds present in a unit volume of oil. The epoxidations were carried out using the iodine value data to measure the unsaturation in oils. Further, the iodine values were used to also find the percentage epoxidation. 


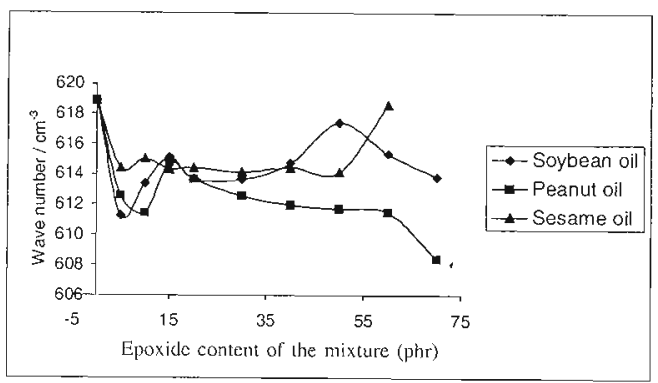

Figure 8: Change in position of the $(\mathrm{C}-\mathrm{Cl})$ position of PVC.

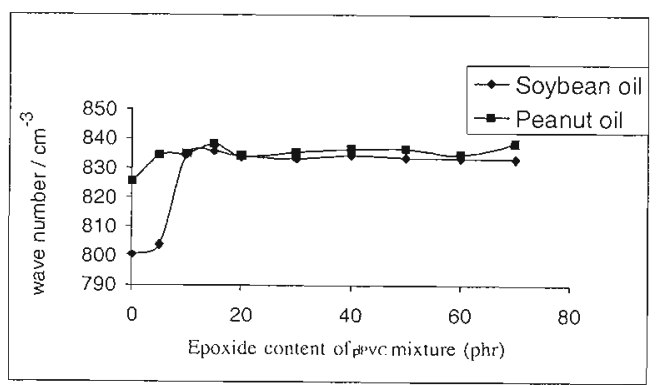

Figure 9: Epoxide interaction.

\section{Confirmation of epoxide}

According to the FT-IR results, there is some evidence that epoxidation has occured. However, the formation of epoxide could not be confirmed as the peaks which appeared in the spectra were not clear. Therefore, as suggested in the literature ${ }^{16,18},{ }^{1} \mathrm{H}$ NMR and ${ }^{13} \mathrm{C}$ NMR analysis were carried out to confirm epoxidation.

\section{${ }^{13} \mathrm{C}$ NMR of epoxidized soybean oil}

The ${ }^{13} \mathrm{C}$ NMR spectrum given in Figure 1 shows a peak at $54 \mathrm{ppm}$, which results from the di-substituted epoxy ring carbon of the epoxidized soybean oil. The absence of a peak around $130 \mathrm{ppm}$ due to di-substituted alkene carbon confirms that the alkene double bond has epoxidized completely.

\section{Analysis of the ${ }^{1} \mathrm{H}$ NMR spectrum}

The peak between $4.0-4.5 \mathrm{ppm}$ as shown in the Figure 2 results from the epoxy-ring proton. This can be confirmed further by analyzing the splitting pattern of the peak in Figure 3. This splitting is caused by cisepoxide. The integration of the peak shows 2 , indicating the number of $\mathrm{H}$-atoms participating in the peak. This corresponds to the two $\mathrm{H}$-atoms in the epoxy ring. ${ }^{18}$

According to the NMR spectral data the formation of epoxidized derivatives of the oils during the epoxidation reaction can be confirmed.

\section{Determination of the epoxidation extent}

According to the spectral data, it can be assumed that the formation of oxiran rings occurs at the expense of double bonds. Since the iodine value is a measure of the amount of double bonds present, the percentage reduction of iodine value in the oil is due to formation of the epoxides. The percentage of epoxydation calculated for each oil for two different levels (50\% and $100 \%$ ) are given in Table 1 and it can be observed that the expected levels of epoxidation were closely achieved.

FT-IR analysis of interaction of pure oils with PVC Changes in the spectral position of the $(\mathrm{C}=\mathrm{O})$ stretching peaks of the pure oils with different amounts in PVC are shown (Figure 4). According to the plot, all three oils have changed their $(\mathrm{C}=\mathrm{O})$ stretching frequencies to lower values when compounded with PVC. This indicates that an interaction has take place through the $\mathrm{C}=\mathrm{O}$ bond of the ester when the oils were mixed with PVC. ${ }^{17}$ Further, analysis of the graph shows that the interaction through the $\mathrm{C}=\mathrm{O}$ bond was at maximum near the 5 phr, 5 phr and 50 phr contents for soybean oil, peanut oil and sesame oil, respectively. However, the further increase of oil contents has led to reduction of the interactions. In addition, the curve for sesame oil has shown another minimum at 5 phr where there is a weaker interaction than the interaction of $\mathrm{CO}$ at $50 \mathrm{phr}$. Another observation is that the interaction in sesame oil is much higher than in the reference soybean oil.

According to the interaction in PVC shown in Figure 5 , the $(\mathrm{C}-\mathrm{Cl})$ bond has also participated in the inter-molecular interactions in the mixture, because the peak position of the (C-Cl) bond has also changed when mixed with the oil. This data has proved that an inter molecular interaction occured between the oil and PVC when the mixture was formed.

The changing patterns of the $(\mathrm{C}-\mathrm{Cl})$ and $(\mathrm{C}=\mathrm{O})$ stretching are not similar. This indicates interaction(s) between oil and PVC in the mixture. There are two possible interactions. One is interaction through the $\mathrm{H}$ of the PVC with $(\mathrm{C}=\mathrm{O})$ of oil as shown (Figure 6 ).

FT-IR spectra of the $100 \%$ epoxidiszed oil derivatives According to the peak positions seen (Figure 7), interactions occured in the PVC mixtures, through the $(\mathrm{C}=\mathrm{O})$ of the epoxidized ester because their positions have shifted towards lower values with the increase of the level of epoxidised ester. Among the three oil derivatives sesame oil derivative has shown the best interaction with PVC. 
As in the case of pure oils, the changing patterns of the position of the two peaks, i.e. $\mathrm{CO}$ and $\mathrm{CCl}$, are different (Figures $7 \& 8$ ). In this case there is an additional polar group, which can interact with PVC. The epoxyring oxygen interacts with the $\alpha-H$ of the PVC and the epoxy-ring carbon atom with the $\mathrm{Cl}$ of the PVC. In order to study the proposed interaction of the epoxy ring the corresponding peak positions must be studied. But the only uninterrupted and clear peak in all cases is the peak located around $830 \mathrm{~cm}^{-1}$. The positional change is shown for peanut and soybean oil derivatives in Figure 9. According to the graph there is an increase in the position of this peak. This implies that the bond responsible for this peak has become stronger. Hence the new interaction of epoxide with PVC is weaker than the interaction that has existed in the epoxide itself.

According to the above evidence, interactions exist between PVC and oil or epoxide in the mixture. These interactions have occured between two polymer chains. Therefore the polymer-polymer interactions must be broken for the oil or epoxide molecule to be inserted between two polymer chains as shown (Figure 10).

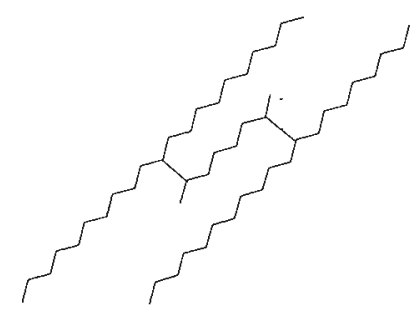

Figure 10: Insertion of a small plasticizer molecule between two polymer chains

Due to this insertion the free volume of the polymer has increased and therefore the freedom of the polymer chains to flow has increased than in the pure PVC. Due to the flow of the polymer chains in the freely available space, the PVC becomes a flexible material. This implies that the sesame oil, peanut oil and their epoxides may impart better flow properties and flexibility to PVC. Sesame oil can interact with PVC much better than soybean oil at higher concentrations. The formation of interactions between the PVC and oils or epoxides enhances the miscibility of the oils or epoxide in PVC.

\section{CONCLUSION}

The sesame oil and peanut oil and their epoxy derivatives interact significantly with PVC molecules upon mixing. These mixtures were compared with epoxidised soybean oil which is used as a plasticizer for PVC. Sesame oil interacts with PVC better than soybean oil. Further, peanut oil and soybean oil show better molecular interactions below 10 phr level, while sesame oil shows maximum interactions around 50 phr.

\section{References}

1. Belhaneche-Bensemra N., Zeddam C. \& Ouahmed S. (2002). Study of the migration of additives from plasticized PVC. Macromolecular Symposium 180: 191-201.

2. Huang J. \& Deanin R.D. (2004). Concentration dependency of interaction parameter between PVC and plasticizers using Inverse Gas Chromatography. Joumal of Applied Polymer Science 91: 146-156.

3. Thomas N.L. (2004). Alloying of poly(vinyl chloride) to reduce plasticizermigration. Journal of Applied Polymer Science 94: 2022-2031.

4. Audic J.L., Reyx D. \& Bross J.C. (2003). Migration of additives from food grade PVC films: Effect of plasticization by polymeric modifiers instead of conventional plasticizers. Joumal of Applied Polymer Science 89: 1291-1299.

5. Ishiaku U.S., Shaharum A., Ismail H. \& Mohd Ishak Z.A. (1997). The effect of an epoxidized plasticizer on the thermooxidative ageing of PVC/ENR thermoplastic elastomer. Polymer International 45: 83-91

6. Gan L.H., Oii K.S., Goh S.H., Gan L.M. \& Leong Y.C. (1995). Epoxidized esters of palm olein as plasticizer for PVC. European Polymer Journal 31(8): 719-724.

7. Simon M.A. \& Kusy R.P. (1996). Plasticizer-level study of poly(vinyl chloride) ion selective membrane. Journal of Biomedical Material Research 30: 313-320.

8. Whelmann J. (1999). Use of esterified rapeseed oil as plasticizer in plastic processing. Fett/Lipid. 101 (6): 249-256.

9. Peter Bredereck (1986). Barium/cadmium for the stabilization of rigid vinyl for exterior applications. Joumal of Vinyl and Additive Technology 8(2): 46-48.

10. Baltaciodlu H. \& Balköse D. (1999). Effect of Zinc Stearate and/or epoxidized soybean oil on gelation and thermal stability of PVC-DOP plastigel. Journal of Applied Polymer Science 74: 2488-2498.

11. Summers J. W. (1981). The nature of poly (vinyl chloride) crystallinity - the microdomain structure. Journal of Vinyl and Additive Technology 3(2): 107-110.

12. Theodorou M. \& Jasse B. (1983). Fourier-transform infrared study of conformational changes in plasticized poly(vinyl chloride). Journal of Polymer Science : Polymer Physics Edition 21 (11): 2263-2274.

13. Afshari A., Gunnarsen P., Clausen A. \& Hansen V. (2004). Emission of phthalates from PVC and other materials. Indoor Air 14(2): 120- 135 .

14. Aboutaybi A., Bouzon A. J. \& Vergnaud J. M. (1990). Study of the preparation of plasticized PVC with a surface with barrier properties. Journal of Vinyl and Additive Technology 12(2): 58-64

15. Feldman D., Banu D., Manley R. St.J. \& Zhu H. (2003). Highly filled blends of a vinyl copolymer with plasticized lignin: Thermal and mechanical properties. Joumal of Applied Polymer Science 89: 2000-2010.

16. Itoi Y., Inoue M. \& Enamoto S. (1986). Epoxidation of fatty acid esters with aqueous hydrogen peroxide in the presence of molybdenum oxide-tributyltin chloride on charcoal catalyst. Bulletin of Chemical Society of Japan 59: 3941-3943.

17. Koening J.L. (1983). Fourier transform Infrared spectroscopy of polymers. Advances in Polymer Science 54: 88-139.

18. Guillén M.D. \& Ruiz A. (2004). Formation of hydroperoxyand hydroxyalkenals during thermal oxidative degradation of sesame oil monitored by proton NMR. European Journal of Lipid Science and Technology 106: 680-687. 